\title{
A consulta telefónica em sete passos
}

Rui Lobo,* Eurico Silva*

\section{RESUMO}

Em todo o mundo a prática de consulta telefónica encontra-se em forte expansão e existe boa evidência de que estas consultas, quando indicadas para a situação visada e adequadas aos objetivos pretendidos, proporcionam níveis elevados de satisfação dos utentes e de eficácia clínica. Entre outras vantagens, as consultas telefónicas permitem aumentar a acessibilidade dos utentes e reduzir custos e inconvenientes da deslocação das populações à sua unidade de saúde. Até há pouco tempo não havia nos cuidados de saúde primários em Portugal muita experiência no uso deste tipo de consultas e a sua prática encontra-se ainda associada a algumas limitações. Os autores propõem algumas estratégias, como a estruturação da consulta telefónica em sete passos e a otimização dos registos clínicos como ferramenta importante para aumentar a sua eficácia e eficiência, rentabilizando o tempo dos profissionais de saúde envolvidos e fazendo destas um bom complemento à atividade assistencial presencial.

Palavras-chave: Consulta telefónica; Medicina geral e familiar; Formação.

\section{INTRODUÇÃO}

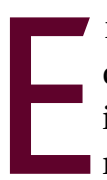

m todo o mundo a prática de telemedicina encontra-se em forte expansão, agora ainda mais impulsionada pelo surgimento das recentes limitações à prática clínica impostas pela pandemia do COVID-19. Não sendo a telemedicina uma novidade no contexto dos cuidados de saúde primários - até agora era usada principalmente como forma de comunicação e troca de pareceres com clínicos de outras especialidades - foi em 2020 que se verificou a necessidade de explorar outros potenciais. Uma das vertentes da telemedicina que mais cresceu nos últimos meses foi precisamente a consulta telefónica, um ato médico em que o clínico efetua uma chamada telefónica ao seu utente e que serve para abordar muitos dos assuntos que seriam tratados numa consulta presencial. Em países como o Reino Unido até um quarto das interações médico-doente nos cuidados de saúde primários são já por telefone. ${ }^{1}$ Entre outras vantagens, as consultas telefónicas têm o potencial de aumentar a acessibilidade dos utentes, reduzir as listas de espera para consulta com o próprio médico, bem como os custos e inconvenientes de deslocação das populações até ao seu centro de saúde. Estudos alargados e com bom nível de evidência demonstram a não inferioridade da satisfação dos utentes com as consultas telefónicas comparativamente às consultas presenciais. ${ }^{2-3}$ No entanto, as consultas telefónicas não são desprovidas de algumas limitações, nomeadamente no que diz respeito ao tempo consumido aos médicos de família face à alternativa tradicional, havendo resultados contraditórios na literatura. ${ }^{4}$

\section{CONTEXTOS CLÍNICOS PASSÍVEIS DE CONSULTA TELEFÓNICA}

A consulta telefónica ao nível da medicina geral e familiar pode ser implementada em diversas situações de doença e vigilância clínica, nas quais existe já uma considerável experiência internacional que suporta o seu uso com segurança e eficácia. A consulta pode ser realizada por iniciativa do utente, por motivo de doença aguda ou necessidade de gestão de problemas de saúde crónicos; ou por iniciativa do profissional de saúde. Neste último caso pode ser útil para avaliar a evolução de um tratamento instituído ou para situações de consulta programada no âmbito de patologias que requerem seguimento ativo, como a hipertensão arterial, diabetes mellitus ou doenças respiratórias crónicas.

Outros contextos clínicos que justificam esta modalidade de consulta são aqueles em que, por risco de

\footnotetext{
*USF João Semana.
} 
doença infetocontagiosa, como a COVID-19, seja recomendável o seguimento destes utentes à distância para maior segurança do paciente e do profissional de saúde. São exemplos casos em que apenas seja necessária a emissão de documentos e certificados médicos que não exijam a avaliação em presença física ou a renovação da prescrição de terapêutica medicamentosa, acompanhamento e suporte dos utentes com quadros depressivos, utentes que se encontrem a realizar cessação tabágica ou situações de doença aguda, como infeções do trato urinário em mulheres, e que, pela sua frequência e simplicidade de tratamento, podem ser orientadas com segurança pelo telefone. ${ }^{5}$

Outro exemplo de uso de consultas telefónicas são os sistemas de triagem telefónica. Não sendo esta uma atividade habitualmente afeta às competências da medicina geral e familiar, pode ser um complemento importante numa altura em que é necessário limitar o fluxo de utentes no interior das instalações da unidade de saúde, ajudando assim a esclarecer antecipadamente, à chegada do utente, a prioridade clínica de atendimento ou a necessidade de redirecionar para outros circuitos de atendimento aqueles utentes que se dirigiam à sua unidade de saúde por outras questões que não por doença aguda. Um dos métodos mais amplamente utilizados consiste numa avaliação telefónica inicial conduzida por uma enfermeira, que orienta, em função da necessidade, para uma consulta médica presencial, para uma consulta médica por telefone ou resolve a situação, se estiver dentro do seu domínio de competências. Diversos estudos têm demonstrado uma maior eficácia na triagem e eficiência na gestão do tempo que estas tarefas consomem aos profissionais. $\mathrm{O}$ estudo recentemente conduzido por Graversen ${ }^{3}$ nos cuidados de saúde primários da Dinamarca comparou várias variáveis de qualidade na consulta telefónica entre diferentes grupos profissionais (enfermeiros, médicos de medicina geral e familiar e médicos de outras especialidades) e demonstrou a não inferioridade na eficácia da triagem e na satisfação dos utentes quando esta abordagem telefónica inicial é conduzida pela equipa de enfermagem comparativamente aos médicos de medicina geral e familiar e demonstraram superioridade em relação ao grupo constituído por médicos de outras especialidades.
Se, por um lado, se estima que cerca de dois terços das solicitações dos utentes nos cuidados de saúde primários podem ser resolvidas por telefone, sem necessidade de agendar nova consulta presencial a curto prazo, e estas consultas são em média 4-6 minutos mais breves do que as consultas presenciais, é no trabalho de marcação da consulta ou convocatória do utente, preparação da consulta e estabelecimento da conexão que se consome mais tempo. ${ }^{1-2}$

\section{MEIOS DE COMUNICAÇÃO NA CONSULTA TELEFÓNICA}

Quanto à escolha da plataforma ou meio de comunicação com o utente, esta deve ser individualizada e sempre adaptada às necessidades e capacidades do utente. O telefone, pelo seu uso generalizado e ampla disponibilidade, constitui atualmente a ferramenta mais útil na telemedicina. Além do telefone, existem outros meios que reúnem cada vez mais aceitação por parte da população. Entre eles, o $e$-mail prefigura uma ferramenta útil, não só para colocação de questões por parte do utente ao médico que, de outra forma, em muitos casos seriam motivo de marcação de consulta, mas também para envio de resultados de meios complementares de diagnóstico e para obtenção de feed$b a c k$, por exemplo após a introdução de uma nova terapêutica ou a avaliação e orientação de uma situação de doença aguda. O facto de a comunicação escrita por $e$-mail ficar registada pode também vir a ser uma vantagem deste meio, apesar da necessidade de serem ainda estabelecidos procedimentos standard para efetuar os registos da comunicação desta forma.

Outros meios de comunicação como os associados a redes sociais ou novas plataformas de mensagens instantâneas, como o WhatsApp ou o Messenger, também têm vindo a ser frequentemente mais utilizados, ainda que com a grande limitação de imporem uma forte componente de informalidade, o que levanta inúmeras questões de cariz legal.

Em Portugal, no Serviço Nacional de Saúde a experiência no uso de vídeo nas consultas telefónicas é ainda escassa. A videochamada acrescenta sem dúvida valor à consulta e pode dar informações preciosas que permitam a tomada de decisões com maior segurança. A plataforma RSE Live está já preparada para esta realidade e esforços significativos de implementação 
foram desenvolvidos sobretudo ao longo dos últimos meses. Ainda assim, esta modalidade enfrenta inúmeros entraves. Desde logo, a iliteracia informática de uma parte significativa da população, a alta complexidade das plataformas e a falta de suporte informático físico e conexão de Internet eficaz.

A criação do Centro Nacional de Telesaúde visa precisamente a definição de medidas de ação prática e concretas que alavanquem o desenvolvimento da telessaúde em Portugal. ${ }^{6}$

\section{LIMITAÇÕES DA CONSULTA TELEFÓNICA}

Por vezes não se figura viável a execução da consulta telefónica. A falta de informação visual e a perda de parte significativa da comunicação não verbal podem prejudicar gravemente a qualidade da observação clínica. Ainda assim, alguns aspetos da comunicação não verbal podem ser preservados, como o tom de voz, a hesitação e a fluidez do discurso.

Igualmente deve ser privilegiada a realização de consulta presencial em detrimento da consulta telefónica sempre que surjam situações em que o exame físico seja imprescindível ou que os utentes sejam incapazes de efetuar a automonitorização de algum sinal ou sintoma. Quando a capacidade comunicativa do utente for reduzida e este não tiver ninguém que o apoie, dever-se-á optar por conversar presencialmente com ele de forma a assegurar que a mensagem é corretamente entendida.

A consulta telefónica tende a limitar a atenção aos sinais e sintomas apresentados pelo doente e frequentemente os utentes não são avaliados de forma tão abrangente pela perda de informação não verbal. ${ }^{7}$

\section{A CONSULTA TELEFÓNICA EM SETE PASSOS}

Alguns passos definidos de consulta telefónica podem ser seguidos de modo a estruturar a intervenção médica e conseguir maiores taxas de sucesso terapêutico, maior conforto e segurança dos profissionais e satisfação dos utentes com os cuidados prestados. ${ }^{8}$

Adaptados da consulta estruturada em três fases, subdivididas em sete passos, proposta por Ramos, ${ }^{9}$ os sete passos da consulta telefónica elencados na Tabela 1 são uma proposta de organização de uma consulta telefónica estruturada.

Os passos dois (estabelecer confiança), quatro (esclarecimento) e cinco (ação) são cruciais para o suces- so da intervenção médica por telefone. Se, por um lado, o estabelecimento de confiança, no passo dois, pode ser dificultado pelo distanciamento físico, um conhecimento prévio das características do utente e uma relação médico-doente bem aprofundada pode facilitar esta etapa. Além de se identificar inequivocamente, o médico deve em seguida procurar adequar o tom de voz de forma a transmitir exatamente a mensagem que pretende.

Outra etapa facilmente comprometida pela abordagem telefónica prende-se com o erro na interpretação e compreensão por parte do utente. Desta forma, é importante sumarizar e reformular a informação obtida, ao nível do passo quatro, e confirmar que o plano acordado foi bem compreendido, ao nível do passo cinco.

Desta forma, estão reunidas as condições para a realização de consultas médicas de qualidade pelo menos equivalente às consultas presenciais tradicionais.

\section{A RELAÇÃO MÉDICO-DOENTE NESTE CONTEXTO}

Quando se tenciona prestar cuidados médicos aos utentes através do telefone é necessário ter em conta as dificuldades acrescidas em estabelecer ou mesmo manter a relação médico-doente. Idealmente deve já existir uma ligação de confiança estabelecida e o sucesso desta abordagem será tanto maior quanto mais duradoura e profunda for esta relação. De forma a maximizar a qualidade e a continuidade do seguimento dos utentes, algumas organizações, como o The Royal Australian College of General Practitioners, sugerem mesmo que os utentes elegíveis para consulta telefónica sejam aqueles que visitaram presencialmente a sua unidade de saúde e contactaram com o seu médico de família pelo menos uma vez ao longo dos últimos 12 meses..$^{10}$ Assim, são de evitar primeiras consultas e observações médicas após um longo período de ausência de prestação de cuidados, exceto quando as circunstâncias assim o obriguem; pese embora o facto de ser possível ao médico a consulta do processo clínico completo do utente, através dos registos no Registo de Saúde Eletrónico (RSE), o que permite um conhecimento de grande parte do historial do utente e, em caso de necessidade, pode ajudar a mitigar o efeito de falta de confiança prévia e relação terapêutica. O conhecimento prévio da história médica passada do utente, não só torna a consulta mais 
TABELA 1. Os sete passos da consulta telefónica estruturada, adaptados de Ramos ${ }^{9}$

\begin{tabular}{|c|c|}
\hline \multirow[t]{2}{*}{ Fase inicial } & $\begin{array}{l}\text { Passo 1. Preparação } \\
\text { - Reunir toda a informação sobre o utente. } \\
\text { - Prever possíveis atrasos durante a consulta telefónica. } \\
\text { - Tomar notas. }\end{array}$ \\
\hline & $\begin{array}{l}\text { Passo 2. Estabelecer confiança } \\
\text { - Identificar-se perante o utente. } \\
\text { - Adequar o tom de voz. } \\
\text { - Reconhecimento das emoções do utente. } \\
\text { - Reconhecimento da experiência prévia do utente com os serviços de saúde. } \\
\text { - Demonstrar empatia ao mostrar que o utente está a ser ouvido. }\end{array}$ \\
\hline \multirow[t]{3}{*}{ Fase intermédia } & $\begin{array}{l}\text { Passo 3. Exploração } \\
\text { - Questionar escolhendo perguntas abertas ou fechadas. } \\
\text { - Explorar a situação através da reflexão. }\end{array}$ \\
\hline & $\begin{array}{l}\text { Passo 4. Avaliação } \\
\text { - Permitir que o utente se expresse, respeitando silêncios. } \\
\text { - O conhecimento que o utente tem sobre o assunto. } \\
\text { - Reflexão. } \\
\text { - Sumarizar e reformular a informação obtida. }\end{array}$ \\
\hline & $\begin{array}{l}\text { Passo 5. Plano de ação } \\
\text { - Encorajar e capacitar o utente a tomar medidas, quando possível. } \\
\text { - Explicar que ação se vai desenvolver para benefício do utente. } \\
\text { - Negociar e confirmar que o plano acordado foi bem compreendido. }\end{array}$ \\
\hline \multirow[t]{2}{*}{ Fase final } & $\begin{array}{l}\text { Passo 6. Final / Encerramento } \\
\text { - Momento em que o utente sente que foi ouvido, respeitado e compreendido, e os problemas que } \\
\text { motivaram a consulta foram todos abordados. } \\
\text { - A chamada termina para ambos, mas a consulta continua para o médico. }\end{array}$ \\
\hline & $\begin{array}{l}\text { Passo 7. Após a chamada/Resumo } \\
\text { - Completar registo clínico/notas. } \\
\text { - Tempo para refletir. } \\
\text { - Tomar outras ações. }\end{array}$ \\
\hline
\end{tabular}

confortável para ambas as partes envolvidas, como a torna também mais segura do ponto de vista da boa prática médica.

Não raramente, os médicos têm sido alvo de queixas de má prática médica e esta modalidade emergente de consultas tem potencial para aumentar o número de casos destas situações. Importa, por isso, ser especialmente cuidadoso na elaboração de bons registos clíni- cos que não só documentem bem todas as interações médico-doente, mas que salvaguardem também o clínico perante problemas legais que possam surgir no contexto das consultas telefónicas.

Ao longo da sua formação, os médicos, sobretudo aqueles com especialidades intensamente relacionais, como a medicina geral e familiar, contactam com dezenas de outros médicos dos quais absorvem 
diferentes estilos e posturas em consulta, bem como diferentes técnicas de comunicação. Cada um forma uma noção daquilo que julga ser mais correto e desejável para a sua prática clínica, assim como atitudes e procedimentos a evitar em contexto de consulta. Há, portanto, um grande número de termos comparativos e uma vasta possibilidade de autoavaliação do modelo e estilo de consulta presencial de cada médico. Quando se transpõe esta análise para a realidade de consulta telefónica, o mesmo não se verifica. Poucos profissionais tiveram na sua formação médica algum contacto teórico ou prático respeitante às técnicas de abordagem e comunicação com utentes pelo telefone. Assim, torna-se imprescindível a formação específica nesta área, bem como a partilha de experiência entre profissionais como forma de benchmarking no seio da comunidade médica.

\section{REGISTOS CLÍNICOS DA CONSULTA TELEFÓNICA}

À semelhança do que acontece nas consultas presenciais, o registo clínico das consultas telefónicas pode seguir o formato do SOAP.

No campo «S» importa identificar inequivocamente, e logo em primeiro lugar, que se trata de uma consulta telefónica; o que pode alterar a interpretação de toda a informação que se segue. Neste ponto continuam a ser registados as queixas e os sentimentos do utente.

Ao nível do campo «O» poder-se-á igualmente registar resultados de análises e outros meios complementares de diagnóstico, bem como a descrição de lesões documentadas pelo utente e enviadas ao médico em suporte digital, como uma tumefação de alguma região ou alteração cutânea. Aqui pode-se pedir o envio por email de fotografia da lesão, permitindo assim inclusivamente o seguimento da evolução da mesma pelo paciente e pelo médico numa posterior consulta telefónica. Dados simples e objetivos do exame físico, como o peso, a tensão arterial ou a temperatura corporal podem ser inquiridos e registados aqui.

O campo «A» continua a ser a avaliação clínica integrada feita pelo médico, não sofrendo alterações significativas. Apenas de assinalar que pode ser mais difícil estabelecer diagnósticos confirmados, em função de a consulta não ser presencial.

Já no campo «P», além de registar todo o plano de seguimento e a orientação acordada com o utente na sequência da decisão partilhada, é de particular interesse deixar estipulado a rede de apoio à disposição do utente em caso de evolução desfavorável, isto é, informar que os canais de comunicação estão abertos e à sua disposição e que, em caso de necessidade, será agendada nova consulta telefónica ou presencial. Explicar sinais de alarme e alterações que devem motivar agendamento de nova consulta telefónica ou de consulta presencial.

No futuro seria interessante ter a capacidade de integrar mais facilmente todos os meios de comunicação e o material de relevo, como emails com documentos fornecidos pelo utente, numa só plataforma de forma a concentrar a informação, melhorar a acessibilidade e facilidade de consulta a todos os profissionais de saúde.

\section{ESTRATÉGIAS PARA OTIMIZAÇÃO DA CONSULTA TELEFÓNICA}

De forma a tornar o processo da consulta telefónica mais eficiente, poupador de tempo e mais confortável para os utentes, algumas estratégias podem ser implementadas.

Desde logo, a preparação da consulta telefónica; quando se tratar de consulta programada, esta pode ser previamente agendada pelo secretário clínico ou enfermeiro que contacta o utente, por telefone ou email e explica como irá decorrer o procedimento, preparando o utente para receber a chamada do médico no dia e hora agendados. Assim, diminui-se o risco de o utente não atender e de se perder essa oportunidade de consulta. Também nesta ocasião o profissional que faz o contacto inicial pode instruir o utente a fazer chegar à sua unidade de saúde exames complementares de diagnóstico, folhas de automonitorização de tensão arterial ou glicemias, bem como outros documentos de relevo para a consulta médica. Esta entrega por parte do utente pode igualmente ser feita por email ou presencialmente, deixando os mesmos na secretaria da unidade de saúde. Outra alternativa cada vez mais usada é a disponibilização dos resultados dos meios complementares de diagnóstico online, dispensando o utente de ter de se deslocar ao laboratório e de tomar diligências para fazer chegar os resultados ao seu médico. Assim, no dia e hora da consulta, e sem perder mais tempo, o médico já vai dispor de todo o material necessário para fazer uma boa consulta. 
No dia da consulta, à hora agendada e após ser efetivada, a chamada pode ser iniciada por outro profissional de saúde que não o médico. Quando a conexão estiver estabelecida, a chamada é passada ao médico que inicia a consulta. Mais uma vez, esta medida, apesar de necessitar de uma estrutura organizada bem montada, permite racionalizar o tempo que o clínico tem para fazer as consultas telefónicas.

Durante a consulta telefónica, o médico tem de ser capaz de efetuar registos clínicos de qualidade enquanto ouve e avalia as queixas do utente. Para isso, o uso de auriculares ou de «modo alta-voz» permite libertar as mãos e realizar simultaneamente a consulta do processo clínico, os registos clínicos, a emissão de credenciais para meios complementares de diagnóstico e de prescrições medicamentosas.

Seguidamente, estes documentos gerados no decorrer da consulta podem ser enviados de forma eletrónica, por mensagem de texto ou email para os utentes. $\mathrm{O}$ médico pode ainda confirmar em tempo real que o utente os recebeu no telemóvel ou na caixa de email.

\section{CONCLUSÃO}

Sem dúvida que a consulta telefónica é uma modalidade de prática clínica eficiente, segura, promissora, cada vez mais usada e que, pelas suas vantagens associadas e pela necessidade de reduzir o número de consultas presenciais, veio para ficar. É um bom complemento à atividade assistencial nos cuidados de saúde primários, sobretudo quando há uma relação médicodoente já bem estabelecida, não substituindo por completo a consulta presencial, que continua a ser o gold-standard.

No entanto, continua a ter algumas limitações associadas, como a falta da componente não verbal da comunicação ou a impossibilidade de se realizar um exame físico completo. Para que o seu uso possa ser mais abrangente e generalizado requer mais investimento, quer ao nível de infraestrutura e suporte técnico quer ao nível da formação e capacitação dos profissionais e dos utentes.

O modelo da consulta telefónica em sete passos e as estratégias apontadas para otimizar a sua aplicação podem ser ferramentas importantes para a melhoria da qualidade e uniformização deste procedimento entre os profissionais de saúde.

\section{REFERÊNCIAS BIBLIOGRÁFICAS}

1. National Health Service. 10 High impact actions. 2.1. Topic sheet: phone consultation [homepage]. London: NHS; 2020. Available from: https://www.networks.nhs.uk/nhs-networks/releasing-capacity-in-general-practice/documents/2-1-topic-sheet-phone-consultations/ show_all_similar_networks

2. Hewitt H, Gafaranga J, McKinstry B. Comparison of face-to-face and telephone consultations in primary care: qualitative analysis. $\mathrm{Br} \mathrm{J}$ Gen Pract. 2010;60(574):e201-12.

3. Graversen DS, Huibers L, Christensen MB, Bro F, Christensen HC, Vestergaard $\mathrm{CH}$, et al. Communication quality in telephone triage conducted by general practitioners, nurses or physicians: a quasi-experimental study using the AQTT to assess audio-recorded telephone calls to out-of-hours primary care in Denmark. BMJ Open. 2020;10(3): e033528.

4. Downes MJ, Mervin MC, Byrnes JM, Scuffham PA. Telephone consultations for general practice: a systematic review. Syst Rev. 2017;6(1):128.

5. Rastogi R, Martinez KA, Gupta N, Rood M, Rothberg MB. Management of urinary tract infections in direct to consumer telemedicine. J Gen Intern Med. 2020;35(3):643-8.

6. Centro Nacional de TeleSaúde. CNTS [homepage].Available from: http:// www.cnts.min-saude.pt/

7. Van Galen LS, Car J. Telephone consultations. BMJ. 2018;360:k1047.

8. GP-Training.net. 2020. Stages of the telephone consultations [homepage]. GT-Training.net; 2020. Available from: https://www.gp-training. net/communication-skills/telephone-consultations/stages-of-the-telephone-consultation/

9. Ramos V.A consulta em 7 passos: execução e análise crítica de consultas em medicina geral e familiar [The consultation in 7 steps: performance and review of consultations in family medicine]. Rev Port Med Geral Fam. 2009;25(2):208-20. Portuguese

10. Royal Australian College of General Practitioners. Guide to providing telephone and video consultations in general practice [Internet]. Melbourne: RACGP; 2020 Mar [updated 2020 Apr 6]. Available from: Available from: https://www.racgp.org.au/FSDEDEV/media/documents/ Clinical\%20Resources/Guidelines/Guide-to-providing-telephone-andvideo-consultations.pdf

\section{CONFLITO DE INTERESSES}

Os autores declaram não ter quaisquer conflitos de interesse.

\section{ENDEREÇO PARA CORRESPONDÊNCIA}

Rui Lobo

E-mail: antonioruilobo@gmail.com

http://orcid.org/0000-0002-7460-5550

Recebido em 07-06-2020

Aceite para publicação em 21-09-2020 


\section{ABSTRACT}

\section{TELEPHONE CONSULTATION IN SEVEN STEPS}

Worldwide, the practice of telemedicine is growing exponentially and there is a good level of evidence that these telephone consultations when used in the appropriate situations and adequate to our goals, provide high patient satisfaction and clinical efficacy. Among other advantages, telephone consultations increase the accessibility of users and reduce the costs and inconveniences of having to physically go to the health center. Until recently, there was little experience in primary health care in Portugal in the use of this type of consultation and there are still some limitations associated with this practice. The authors suggest some strategies, such as structuring the telephone consultation in seven steps, here discussed, and the optimization of clinical records, are some of the important tools to increase their effectiveness and to maximize the work time of health professionals involved, making these a good complement to the traditional consultations.

Keywords: Telephone consultation; Family practice; Training. 